\title{
Sphere Representations, Stacked Polytopes, and the Colin de Verdière Number of a Graph
}

\author{
Lon Mitchell \\ Mathematical Reviews \\ American Mathematical Society \\ Ann Arbor, Michigan, U.S.A. \\ Ihm@ams . org
}

\author{
Lynne Yengulalp \\ Department of Mathematics \\ University of Dayton \\ Dayton, Ohio, U.S.A. \\ lyengulalp1@udayton.edu
}

Submitted: Jun 8, 2014; Accepted: Dec 8, 2015; Published: Jan 22, 2016

Mathematics Subject Classifications: 05C50, 05C10

\begin{abstract}
We prove that a $k$-tree can be viewed as a subgraph of a special type of $(k+1)$ tree that corresponds to a stacked polytope and that these "stacked" $(k+1)$-trees admit representations by orthogonal spheres in $\mathbb{R}^{k+1}$. As a result, we derive lower bounds for Colin de Verdière's $\mu$ of complements of partial $k$-trees and prove that $\mu(G)+\mu(\bar{G}) \geqslant|G|-2$ for all chordal $G$.
\end{abstract}

\section{Introduction}

Yves Colin de Verdière's graph invariant $\mu$ is defined as the maximum nullity over a special class of real symmetric matrices [4]. Among its many interesting properties are that $\mu$ is minor monotone and $\mu(G) \leqslant 3$ if and only if $G$ is planar. These properties are collected in a recent survey by László Lovász [17] based on an earlier paper with Hein van der Holst and Alexander Schrijver [28].

Andrew Kotlov, László Lovász, and Santosh Vempala showed close connections between $\mu$ and certain geometric representations of graphs, including vector representations and sphere representations [15]. They also conjectured that $\mu(G)+\mu(\bar{G}) \geqslant|G|-2$ for all graphs $G$. Such an inequality for a graph parameter defined using the maximum nullity over a class of matrices has been called the "graph complement conjecture" for that parameter [1]. Inequalities that bound $p(G)+p(\bar{G})$ or $p(G) p(\bar{G})$ for some parameter $p$ are also called Nordhaus-Gaddum type for the authors of early results on the chromatic number of graphs [22]. Recent examples include $\operatorname{tw}(G)+\operatorname{tw}(\bar{G}) \geqslant|G|-2[7,12]$, where $\operatorname{tw}(G)$ is the tree-width of $G$, and upper bounds involving a multiple of $|G|$ for Colin de Verdière type parameters [2]. Leslie Hogben has written a nice survey of NordhausGaddum problems for Colin de Verdière type parameters, variants of tree-width, and related parameters [10]. 
In this article, we obtain lower bounds for $\mu$ of complements of partial $k$-trees. In particular, we obtain one bound for general $k$-trees and another slightly better one for $k$-trees that correspond to stacked polytopes. The study of these "stacked" $k$-trees is also suggested by recent calculations of $\mu$ for chordal graphs by Shaun Fallat and the first author [8], which in turn were based on similar patterns appearing in the study of $\mu$ for split graphs by Felix Goldberg and Abraham Berman [9]. We use the results and connections established by Kotlov, Lovász, and Vempala in order to prove these lower bounds. Finally, we combine our new lower bounds with the chordal calculations to prove that $\mu(G)+\mu(\bar{G}) \geqslant|G|-2$ for all chordal $G$.

Besides the work already cited, polytopes and sphere and vector representations have featured prominently in other recent work on $\mu$. In particular, Ross Kang, László Lovász, Tobias Müller, and Edward Scheinerman used sphere representations by tangent spheres ${ }^{1}$ (coin representations) and properties of $\mu$ for planar graphs to study dot product representations [13]. As for polytopes, Ivan Izmestiev has studied connections between nullspace representations and skeletons of convex polytopes [11]. In light of those results, perhaps a natural follow-up to our work would be to consider sphere representations and skeletons of polytopes.

In Section 2 we introduce $k$-trees and stacked $k$-trees. In Section 3 we define $\mu$ and detail how $\mu$ is related to vector and sphere representations. In Section 4 we prove our main results by combining sphere representations and stacked $k$-trees.

\section{$2 \quad$ Stacked $k$-Trees}

A clique is a subgraph isomorphic to the complete graph $K_{n}$ on $n$ vertices for some $n$ (note that we do not assume a clique to be maximal). A vertex $v$ of a graph is simplicial if it, together with its neighbors, $N(v)$, form a clique. A graph is chordal if it has no induced cycles of length at least four. All chordal graphs have at least one simplicial vertex [5].

A tree decomposition of a graph $G=(V, E)$ consists of a tree $T$ and a family of subsets of vertices of $G,\left\{X_{i}\right\}$, one for each vertex of $T$, such that

- $\bigcup_{i} X_{i}=V$;

- if $u v \in E$ there exists a vertex $i$ of $T$ such that $u \in X_{i}$ and $v \in X_{i}$; and

- for all vertices $i, j$, and $k$ of $T$, if $j$ is on the path from $i$ to $k$, then $X_{i} \cap X_{k} \subseteq X_{j}$.

The width of a tree decomposition is one less than the size of a largest $X_{i}$. The treewidth $\operatorname{tw}(G)$ of $G$ is the minimum width over all tree decompositions of $G$.

A $k$-tree is a (chordal) graph constructed inductively by starting with a complete graph on $k+1$ vertices and connecting each new vertex to the vertices of an existing clique on $k$ vertices. The maximal cliques of a $k$-tree are all size $k+1$ [24].

\footnotetext{
${ }^{1}$ For interested readers, Lovász also has a helpful survey of many types of geometric graph representations [19] as an update of a paper with Katalin Vesztergombi [20].
} 
A graph that is a subgraph of a $k$-tree is a partial $k$-tree. The treewidth of a graph $G$ can also be characterized as the smallest $k$ such that $G$ is a partial $k$-tree [3].

For vertices $x$ and $y$ of a graph $G$, an $x, y$-separator is a subset $S$ such that $x$ and $y$ are in different connected components of $G-S$. A separator is a subset $S$ of $G$ such that $G-S$ has more connected components than $G$ does. Chordal graphs are those graphs whose minimal separators are cliques [6]. It is straightforward that any minimal $x, y$-separator of a subgraph $S$ of $G$ is contained in a minimal $x, y$-separator of $G$.

Theorem 1 ([25, Theorem 1.1]). A graph $G$ is a $k$-tree if and only if $G$ is connected, $G$ has a $k$-clique but no $k+2$ clique, and every minimal $x, y$-separator of $G$ is a $k$-clique.

A $k$-simplex is a $k$-dimensional convex hull of $k+1$ points. Stacked polytopes are formed by starting from a simplex and then repeatedly gluing simplices onto the faces of the polytope. A $k$-tree is the 1-skeleton of a stacked polytope if and only if no three $(k+1)$-vertex cliques have $k$ vertices in common [14].

If $H$ is a clique on $n$ vertices of a graph $G$, we define the degree of $H$ in $G$ to be the number of distinct $n+1$ cliques of $G$ of which $H$ is a subgraph (note this definition agrees with the usual definition for the degree of a single vertex). A clique of degree one will be called pendant, and a clique of degree zero is maximal. Using these definitions, a $k$-tree is the graph of a stacked polytope if and only if each $k$-clique has degree at most two. We will call a chordal graph with treewidth $k$ stacked if each $k$-clique has degree at most two.

Lemma 2. If $T$ is a stacked $k$-tree, then each clique on at most $k-1$ vertices is contained in at least two pendant $k$-cliques of $T$.

Proof. Fix a value for $k$. We will prove that each $(k-1)$-clique is contained in exactly two pendant $k$-cliques, and the result will follow from the fact that each clique on at most $k-1$ vertices is contained in a $(k-1)$-clique.

The result is true for $K_{k+1}$ since every $k$-clique is pendant. Assume the result is true for all stacked $k$-trees on $n \geqslant k+1$ vertices and let $T$ be a stacked $k$-tree on $n+1$ vertices. Let $v$ be a simplicial vertex of $T$. Then the induction hypothesis applies to $T-v$ and $N(v)$ is the only $k$-clique whose degree differs in $T-v$ and $T$. Also, in $T-v$, each $(k-1)$-clique in $N(v)$ is contained in at least two pendant $k$-cliques, one of which is $N(V)$. In $T$, each $(k-1)$-clique $C$ of $N(v)$ can no longer count $N(v)$ as a pendant $k$-supergraph, however, $C$ is now contained in the pendant $k$-clique $v \cup C$. Note that each $k$-clique of $T$ that contains $v$ is pendant. So, those $(k-1)$-cliques in $T$ that are not subgraphs of $T-v$, contain $v$ and are therefore in two pendant $k$-cliques, namely, $\{v, x\} \cup C$ and $\{v, y\} \cup C$ where $N(V)-C=\{x, y\}$.

Proposition 3. Suppose that $G$ is a chordal graph with $k \leqslant \operatorname{tw}(G) \leqslant k+1$. If $\operatorname{tw}(G)=$ $k+1$, suppose further that $G$ is stacked. Then there exists a stacked $(k+1)$-tree $H$ such that $G$ is a subgraph of $H$.

Proof. Fix a value for $k$. If $G$ has at most $k+2$ vertices, then $H=K_{k+2}$ suffices. Assume next that $G$ has more than $k+2$ vertices and that the result holds for all graphs on fewer vertices. 
If $G$ is a $(k+1)$-tree, we are done. Suppose $G$ is not a $(k+1)$-tree. If $\operatorname{tw}(G)=k$, then since $G$ is chordal, $G$ has a minimal separator on at most $k$ vertices. If $\operatorname{tw}(G)=k+1$, by Theorem $1 G$ has a minimal $x, y$-separator, and thus a minimal separator, on at most $k$ vertices. Thus $G$ can be written as the union of two strictly smaller chordal subgraphs $G_{1}$ and $G_{2}$ where $S=G_{1} \cap G_{2}$ is a clique on at most $k$ vertices and there are no edges between $G_{1}-S$ and $G_{2}-S$ in $G$. By the induction hypothesis, there exist stacked $(k+1)$-trees $H_{1}$ and $H_{2}$ containing $G_{1}$ and $G_{2}$, respectively. Let $S_{1}$ and $S_{2}$ denote the copies of $S$ in $G_{1}$ and $G_{2}$, respectively.

For each $i$, add $k+1-|S|>0$ new vertices to $H_{i}$ in the following way: make each new vertex, by Lemma 2 , adjacent to a pendant $(k+1)$-clique including both $S_{i}$ and the previously added vertices. The result will be a new graph $H_{i}^{\prime}$ with a new pendant $(k+1)$-clique $S_{i}^{\prime}$ consisting of the new vertices and $S_{i}$. Let $H$ be the union of $H_{1}^{\prime}$ and $H_{2}^{\prime}$ along $S_{1}^{\prime}$ and $S_{2}^{\prime}$ preserving the identification of $S_{1}$ and $S_{2}$.

By construction and since $S_{1}^{\prime}$ and $S_{2}^{\prime}$ were pendant, $H$ is a stacked $(k+1)$-tree containing $G$.

A linear $k$-tree is a $k$-tree that is either complete or that has exactly two vertices with degree $k$. The proper pathwidth of a graph $G$ is the smallest $k$ such that $G$ is a subgraph of a linear $k$-tree. One might hope that a result similar to Proposition 3 is true for linear $k$-trees as well. In general, however, $k$-trees (even 1 -trees) can have arbitrarily large proper pathwidth [27], and thus in general cannot be embedded in linear $(k+1)$-trees.

For each $k \geqslant 1$, let $T_{k}$ be the unique infinite $k$-tree where each $k$-clique has degree two.

Proposition 4. Each finite stacked $k$-tree is a subgraph of $T_{k}$.

Proof. The result is clearly true for $K_{k+1}$. Let $G$ be a stacked $k$-tree on $k+2$ or more vertices, and assume the result holds for all such graphs on one fewer vertex. Let $v$ be a simplicial vertex of $G$. By the induction hypothesis, $G-v$ is a subgraph of $T_{k}$. Since $G$ is stacked and $|G|>k+1$, the vertices of $N(v)$ have exactly two common neighbors in $G$ and exactly one common neighbor in $G-v$. Thus the vertex of $T_{k}$ that is adjacent to all the vertices of $N(v)$ but not part of the image of $G-v$ may be identified with $v$.

\section{Vector and Sphere Representations}

In this section, we present $\mu$ and a parameter $\nu$ associated with vector and sphere representations.

For a simple graph (no loops or multiple edges) $G=(V, E)$ on vertices $1,2, \ldots, n$, the Colin de Verdière number of $G, \mu(G)$, is the maximum corank among all symmetric $n \times n$ matrices $M=\left[m_{i j}\right]$ satisfying the following properties:

M1: $m_{i j}<0$ if $i j \in E$ and $m_{i j}=0$ if $i j \notin E$ (diagonal entries are not restricted);

M2: $M$ has exactly one negative eigenvalue; and 
M3: If $X=\left[x_{i j}\right]$ is a symmetric $n \times n$ matrix with zero diagonal such that $x_{i j}=0$ for $i j \in E$ and $M X=0$, then $X=0$.

Condition M3 is often called the Strong Arnold Property or Strong Arnold Hypothesis.

For the same graph $G$, let $\nu(G)$ be the smallest dimension $d$ such that there exists a vector labeling $\left\{u_{i}\right\}_{i \in V}$ in $\mathbb{R}^{d}$ that satisfies the following conditions:

$\mathrm{U} 1$ : for $i \neq j$, the dot product $u_{i} \cdot u_{j}=1$ if $i j \in E$ and $u_{i} \cdot u_{j}<1$ if $i j \notin E$ (so $u_{i} \cdot u_{i}$ is not explicitly restricted); and

U2: if $X=\left[x_{i j}\right]$ is a symmetric $n$-by- $n$ matrix such that $x_{i j}=0$ for $i j \notin E$ and $\sum_{j} x_{i j} u_{j}=0$ for every node $i$, then $X=0$.

We will call such a labeling a vector representation ${ }^{2}$ of $G$ [23], and $\nu$ is related to $\mu$ by the following theorem:

Theorem 5 ([15, Theorem 3.3]). For every graph $G$ different from $\overline{K_{2}}, \nu(\bar{G})=n-\mu(G)-$ 1.

Given $(d-1)$-spheres $S_{1}$ and $S_{2}$ in $\mathbb{R}^{d}$, define the function

$$
a\left(S_{1}, S_{2}\right)=\frac{\rho^{2}-\left(r_{1}+r_{2}\right)^{2}}{\rho^{2}-\left(r_{1}-r_{2}\right)^{2}}
$$

where $r_{1}$ and $r_{2}$ are the respective radii and $\rho$ is the distance between the centers of $S_{1}$ and $S_{2}$. Then $a\left(S_{1}, S_{2}\right)=a\left(S_{2}, S_{1}\right)$, and $a\left(S_{1}, S_{2}\right)$ is preserved by conformal mappings $\mathbb{R}^{d} \rightarrow \mathbb{R}^{d}$. A similar definition for caps in $\mathbb{S}^{d}$ (spheres together with one of the two regions in $\mathbb{S}^{d}$ that they bound) means that $a\left(S_{1}, S_{2}\right)$ is also preserved by conformal mappings $\mathbb{R}^{d} \rightarrow \mathbb{S}^{d}[15$, p. 502].

If $S_{1}$ and $S_{2}$ intersect at an angle $\alpha$, then $\rho^{2}=r_{1}^{2}+r_{2}^{2}-2 r_{1} r_{2} \cos \alpha$. Thus $S_{1}$ and $S_{2}$ are orthogonal spheres, which we will denote by $S_{1} \perp S_{2}$, if and only if $a\left(S_{1}, S_{2}\right)=-1$ if and only if $\rho^{2}=r_{1}^{2}+r_{2}^{2}$.

An orthogonal sphere representation of a graph $G=(V, E)$ in $\mathbb{R}^{d}$ is a labeling of each vertex $i$ with a $(d-1)$-dimensional sphere $S_{i}$ so that, for $i \neq j, a\left(S_{i}, S_{j}\right)=-1$ if $i j \in E$ and $a\left(S_{i}, S_{j}\right)>-1$ if $i j \notin E$. Orthogonal cap representations in $\mathbb{S}^{d}$ are defined similarly, and, by the remark above, given an orthogonal sphere labeling of $G$ in $\mathbb{R}^{d}$, one can project each sphere into $\mathbb{S}^{d}$ (chosen large enough so that no interior of a sphere is mapped to a hemisphere or more) $[15$, p. 503]. Moreover, an orthogonal cap representation of a graph in $\mathbb{S}^{d}$ yields a vector labeling of $G$ in $\mathbb{R}^{d+1}$ by associating each cap with the center of the unique sphere in $\mathbb{R}^{d+1}$ that is orthogonal to the unit sphere and whose intersection with $\mathbb{S}^{d}$ (viewed as the unit sphere) is the cap. Thus

\footnotetext{
${ }^{2}$ This type of vector representation is similar to a dot product representation, which requires a modified form of the first condition $\left(u_{i} \cdot u_{j} \geqslant 1\right.$ if $\left.i j \in E\right)$ but does not require the second condition. Orthogonal vector representations, such as those famously used in Lovász's calculation of the Shannon capacity of the cycle $C_{5}$ [18], are significantly different and are more closely related to the other Colin de Verdière parameter.
} 
Theorem 6 ([15, Lemma 6.2]). If a graph $G$ has an orthogonal sphere representation in $\mathbb{R}^{d}$, then there exists a vector labeling of $G$ in $\mathbb{R}^{d+1}$ satisfying condition $U 1$.

Proposition 7. Let $G$ be a $k$-tree. Suppose that a vector labeling $\left\{u_{i}\right\}$ of $G$ satisfies condition $U 1$ and the vectors corresponding to the vertices of any clique of $G$ are linearly independent. Then $\left\{u_{i}\right\}$ is a vector representation of $G$.

Proof. The result is true for a graph on one vertex as the corresponding vector must be nonzero. Suppose the result holds for $k$-trees on $n$ vertices and let $G$ be a $k$-tree with $n+1$ vertices. Let $X=\left[x_{i j}\right]$ be a symmetric $n$-by- $n$ matrix such that $x_{i j}=0$ for $i j \notin E$ and $\sum_{j} x_{i j} u_{j}=0$ for every vertex $i$. Let $v$ be a simplicial vertex of $G$, so that the neighbors of $v$ form a clique, and thus the vectors corresponding to those vertices are linearly independent by assumption. Therefore,

$$
\sum_{j} x_{v j} u_{j}=\sum_{v j \in E} x_{v j} u_{j}=0
$$

implies that $x_{v j}=0$ for all $j$. Since $X$ is symmetric, both the row and column of $X$ corresponding to $v$ are zero. Applying the induction hypothesis to $G-v,\left\{u_{i}\right\}_{i \neq v}$, and the submatrix of $X$ obtained by deleting the row and column corresponding to $v$, we may conclude that $X=0$, as desired.

Corollary 8. Let $\bar{G}$ be a k-tree other than $K_{2}$. If $\left\{u_{i}\right\}$ is a vector labeling in $\mathbb{R}^{d}$ of $\bar{G}$ satisfying condition $U 1$ such that $\left\|u_{i}\right\|>1$ for each $i$, then $\mu(G) \leqslant n-d-1$.

Proof. The Gram matrix of the $u_{i}$ corresponding to a clique $C$ in $\bar{G}$ can be written as the sum of a positive definite diagonal matrix and the (positive semidefinite) matrix of all ones, thus has full rank, and so the $u_{i}$ corresponding to $C$ are linearly independent. The result now follows from Theorem 6 and Proposition 7.

\section{Main Results}

We are now in a position to bound $\mu$ for complements of $k$-trees by constructing orthogonal sphere representations.

Lemma 9. Let $S_{1}$ and $S_{2}$ be nontrivial $n$-spheres in $\mathbb{R}^{n+1}$ and let $P$ be a copy of $\mathbb{R}^{k}$ in $\mathbb{R}^{n+1}$ for some $k \geqslant 1$ such that $S_{1} \cap P$ and $S_{2} \cap P$ are nontrivial. Suppose that either $S_{1} \perp S_{2}$ or $\left(S_{1} \cap P\right) \perp\left(S_{2} \cap P\right)$. Then $S_{1} \perp S_{2}$ and $\left(S_{1} \cap P\right) \perp\left(S_{2} \cap P\right)$ if and only if $P$ contains either the center of $S_{1}$ or the center of $S_{2}$.

Proof. Let $r_{1}, r_{1}^{\prime}, r_{2}$, and $r_{2}^{\prime}$ and $c_{1}, c_{1}^{\prime}, c_{2}$, and $c_{2}^{\prime}$ be the radii and centers of $S_{1}, S_{1} \cap P, S_{2}$, and $S_{2} \cap P$, respectively. Then $d_{i}=\left\|c_{i}-c_{i}^{\prime}\right\|$ gives the distance from $c_{i}$ to $P$, and we have $r_{i}^{2}=d_{i}^{2}+\left(r_{i}^{\prime}\right)^{2}$ for each $i$. Set $\rho=\left\|c_{1}-c_{2}\right\|$ and $\rho^{\prime}=\left\|c_{1}^{\prime}-c_{2}^{\prime}\right\|$. Then $\rho^{2}=\left(d_{1} \pm d_{2}\right)^{2}+\left(\rho^{\prime}\right)^{2}$ (with \pm depending on how $c_{1}$ and $c_{2}$ relate to $P$ ), so that

$\rho^{2}-r_{1}^{2}-r_{2}^{2}=\left(d_{1} \pm d_{2}\right)^{2}+\left(\rho^{\prime}\right)^{2}-\left(r_{1}^{\prime}\right)^{2}-d_{1}^{2}-\left(r_{2}^{\prime}\right)^{2}-d_{2}^{2}=\left(\rho^{\prime}\right)^{2}-\left(r_{1}^{\prime}\right)^{2}-\left(r_{2}^{\prime}\right)^{2} \pm 2 d_{1} d_{2}$.

Thus if either $S_{1} \perp S_{2}$ or $\left(S_{1} \cap P\right) \perp\left(S_{2} \cap P\right)$, the other can only happen if either $d_{1}$ or $d_{2}$ is zero. 


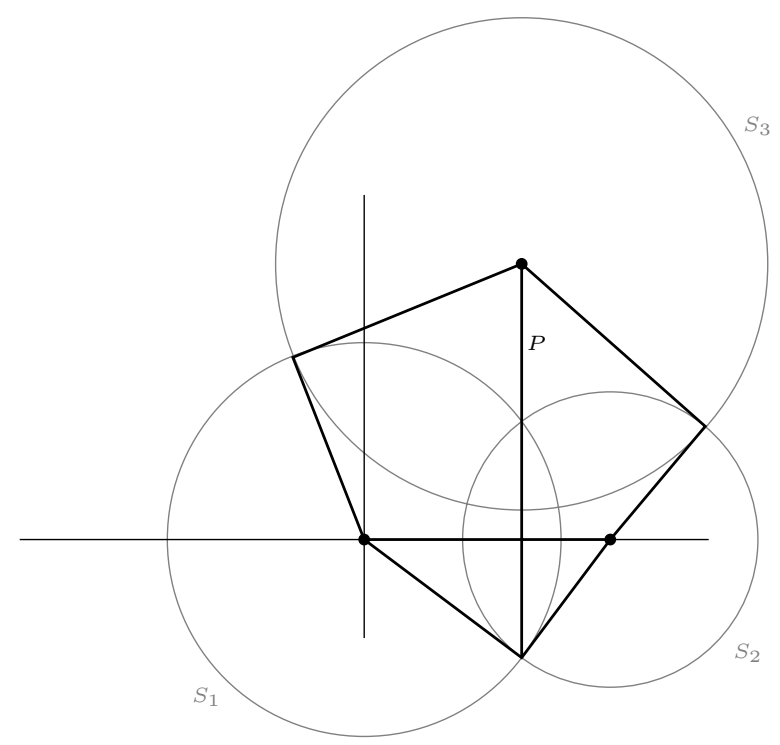

Figure 1: Three orthogonal circles as in Lemma 10.

Lemma 10. Given orthogonal n-spheres $S_{1}$ and $S_{2}$ in $\mathbb{R}^{n+1}, S_{1} \cap S_{2}$ is an $(n-1)$-sphere that lies in a unique $n$-dimensional hyperplane $H$. A third n-sphere $S_{3}$ is orthogonal to both $S_{1}$ and $S_{2}$ if and only if the center of $S_{3}$ lies on $H$ and $S_{1} \cap S_{2}$ and $S_{3} \cap H$ are orthogonal $(n-1)$-spheres.

Proof. Since $S_{1}$ and $S_{2}$ are orthogonal, $S_{1} \cap S_{2}$ is nonempty and does not consist of a single point. Thus $S_{1} \cap S_{2}$ is an $(n-1)$-sphere lying in a unique hyperplane $H$ of dimension $n$. Since $S_{1} \cap H=S_{2} \cap H=S_{1} \cap S_{2}$, the result follows by applying Lemma 9 to $S_{3}$ and $S_{1}$ and to $S_{3}$ and $S_{2}$.

Lemma 11. Given $n$ mutually orthogonal $(n-1)$-spheres $S_{1}, \ldots, S_{n}$ in $\mathbb{R}^{n}, \bigcap_{i} S_{i}$ consists of two points. Let $L$ be the line through those two points and let $U$ be the union of the interiors of the spheres. Given any point $p$ on $L \backslash \mathrm{cl} U$, there exists an $(n-1)$-sphere, $S(p)$, that is orthogonal to each $S_{i}$ and has center $p$. Furthermore, the radius of $S(p)$ approaches 0 as $p$ approaches $U$ along $L$.

Proof. We use induction on $n$. In $\mathbb{R}$, a single 0-sphere consists of two points $c \pm r$, and given any point $p$ outside of the interval $[c-r, c+r]$ there is a positive solution to $r_{1}^{2}=(c-p)^{2}-r^{2}$. Assume the result is true for all $n \leqslant k$ for some $k$, and suppose $n=k+1$. Suppose we have mutually orthogonal $k$-spheres $S_{1}, \ldots, S_{k+1}$ in $\mathbb{R}^{k+1}$. By Lemma 10, $S_{k} \cap S_{k+1}$ defines a hyperplane $H \approx \mathbb{R}^{k}$. For each $i \leqslant k$, let $R_{i}=S_{i} \cap H$. Note that $R_{k}=S_{k} \cap S_{k+1}$ and therefore $\bigcap\left\{R_{i}: i \leqslant k\right\}=\bigcap\left\{S_{j}: j \leqslant k+1\right\}$. Again by Lemma $10, R_{1}, \ldots, R_{k}$ are mutually orthogonal $(k-1)$-spheres in $H$. By the induction hypothesis, $\bigcap\left\{R_{i}: i \leqslant k\right\}$ consists of two points. Let $L$ and $U$ be as in the statement of the Lemma (for the $S_{i}$ ). By definition, $L \subset H$. Let $p \in L \backslash \operatorname{cl} U=L \backslash \operatorname{cl}(U \cap H)$. Again by the induction hypothesis, there exists a $k-1$-sphere $S^{\prime}(p)$ with center at $p$ such that 
$S^{\prime}(p)$ is orthogonal to each $R_{i}$. Let $S(p)$ be the $k$-sphere with the same center and radius as $S^{\prime}(p)$. Denote the radii of $S(p)$ and $S_{1}$ as $r_{p}$ and $r_{1}$ respectively. Let $c_{1}$ be the center of $S_{1}$ and let $\rho_{p}$ be the distance between $p$ and $c_{1}$. Since $S(p)$ and $S_{1}$ are orthogonal $r_{p}^{2}+r_{1}^{2}=\rho_{p}^{2}$. Hence as $p$ approaches $U$ along $L, p$ approaches $S_{1}, \rho_{p}$ approaches $r_{1}$, and therefore $r_{p}$ approaches 0 .

Proposition 12. Each $T_{k}$ has an orthogonal sphere representation in $\mathbb{R}^{k}$ with spheres of positive radius.

Proof. We construct a nested sequence of representations, $\left(\mathcal{S}_{k}^{i}\right)$, of finite subtrees of $T_{k}$ inductively and then we set $\mathcal{S}_{k}=\bigcup_{i=1}^{\infty} \mathcal{S}_{k}^{i}$. Let $T_{k}^{0}=K_{k+1}$ and let $T_{k}^{i+1}$ be the graph obtained from $T_{k}^{i}$ by adding a new vertex to each pendant $k$-clique. Assuming we have defined a representation $\mathcal{S}_{k}^{i}$ for $T_{k}^{i}$, for a vertex $v$ of $T_{k}^{i}$ let $s(v)$ be the corresponding $(k-1)$-sphere in $\mathcal{S}_{k}^{i}$ and let $D(v)$ be the closed $k$-ball with boundary $s(v)$.

Represent $T_{k}^{0}$ by starting with a set, $\mathcal{S}_{k}^{0}$, of $k+1$ mutually orthogonal nontrivial $(k-1)$ spheres in $\mathbb{R}^{k}$. Note that for each pendant $k$-clique $C$ of $T_{k}^{0}$ there exists a point $p(C)$ of $\bigcap_{v \in C} s(v)$ that is not in $\bigcup_{v \notin C} D(v)$. Note also that distinct elements of $\mathcal{S}_{k}^{0}$ are either orthogonal or disjoint.

Assume that we have a representation $\mathcal{S}_{k}^{i}$ for $T_{k}^{i}$ such that for each pendant $k$-clique $C$ of $T_{k}^{i}$ there exists a point $p(C)$ of $\bigcap_{v \in C} s(v)$ that is not in $\bigcup_{v \notin C} D(v)$ and further suppose the elements of $\mathcal{S}_{k}^{i}$ are either orthogonal or disjoint. Then for each pendant clique $C_{j}$ there exists an open neighborhood $U\left(C_{j}\right)$ of $p\left(C_{j}\right)$ that does not intersect $\bigcup_{v \notin C_{j}} D(v)$. We may choose the $U\left(C_{j}\right)$ to be pairwise disjoint. By Lemma 11, for each $j$ choose a new sphere $S_{j}$ inside $U\left(C_{j}\right)$ orthogonal to each element of $\left\{s(v): v \in C_{j}\right\}$. Let $\mathcal{S}_{k}^{i+1}=\mathcal{S}_{k}^{i} \cup\left\{S_{j}\right\}_{j}$.

By construction and by our choices of the $U\left(C_{j}\right), \mathcal{S}_{k}^{i+1}$ represents $T_{k}^{i+1}$, and two distinct elements of $\mathcal{S}_{k}^{i+1}$ are either orthogonal or disjoint. Let $C$ be a pendant $k$-clique of $T_{k}^{i+1}$ and let $w \notin C$ be the additional vertex of the maximal clique containing $C$. By Lemma 11, $\bigcap_{v \in C} s(v)$ consists of two points, one of which, $p(C)$, does not lie in the interior of $D(w)$. By our choice of $U\left(\left(C \cap T_{k}^{i}\right) \cup\{w\}\right), p(C)$ is not in $D(v)$ for any $v$ not in $C$.

Corollary 13. If $G$ is the complement of a partial $k$-tree, then $\mu(G) \geqslant n-k-3$.

Proof. By assumption, $\bar{G}$ is a subgraph of a $k$-tree $\overline{G^{\prime}}$. Thus $\overline{G^{\prime}}$ is a subgraph of a stacked $(k+1)$-tree, $\bar{H}$, by Proposition 3. If $H \neq \bar{K}_{2}$, then $\mu(H)=n-\nu(\bar{H})-1$, by Theorem 5 . If $H=\bar{K}_{2}$, then $\mu(H)=1 \geqslant-2[17$, Example 2.7]. By Proposition 3, Proposition 4, and Proposition $12, \bar{H}$ has an orthogonal sphere representation in $\mathbb{R}^{k+1}$. By Theorem 6 and Proposition $7, \bar{H}$ has a vector representation in $\mathbb{R}^{k+2}$. Thus $\nu(\bar{H}) \leqslant k+2$.

Corollary 14. If $G$ is the complement of a subgraph of a stacked k-tree, then $\mu(G) \geqslant$ $n-k-2$.

Proof. The proof is the same as that of Corollary 13 except now $\bar{H}$ may be assumed to be stacked.

Theorem 15 ([8, Theorem 5.7]). If $G$ is a stacked chordal graph, then $\mu(G)=\operatorname{tw}(G)$. If $G$ is a chordal graph that is not stacked, then $\mu(G)=\operatorname{tw}(G)+1$. 


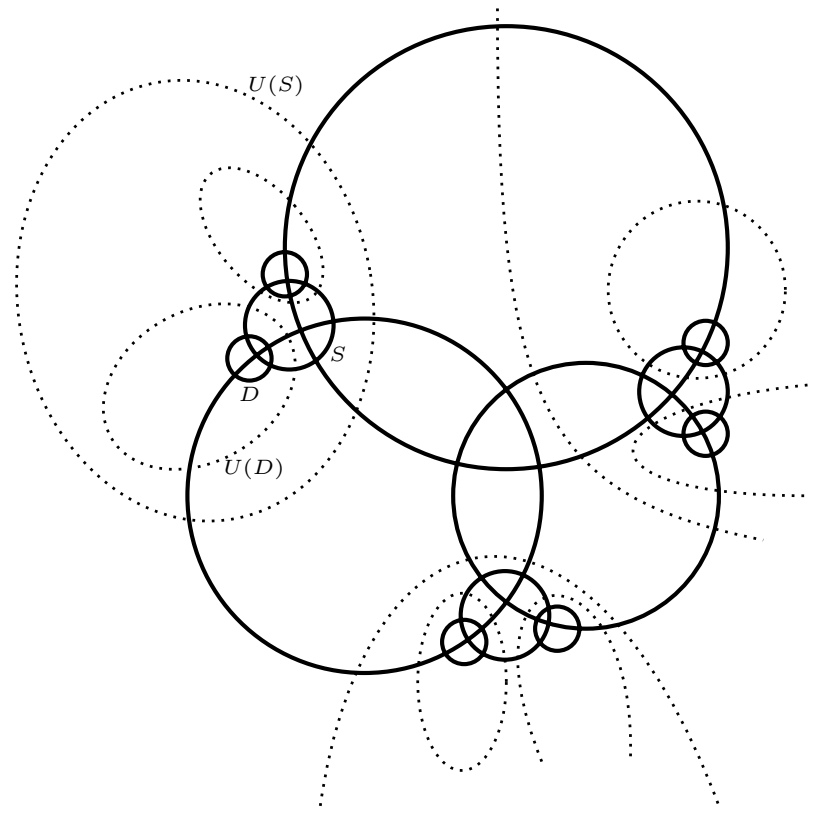

Figure 2: First three stages of construction for $T_{2}$ in Proposition 12.

Proposition 16. For a chordal graph $G, \mu(G)+\mu(\bar{G}) \geqslant n-2$.

Proof. Let $\operatorname{tw}(G)=k$. Then $G$ is a partial $k$-tree. If $G$ is stacked, then $\mu(G)=k$ by Theorem 15 and $\mu(\bar{G}) \geqslant n-k-2$ by Corollary 14 . If $G$ is not stacked, then $\mu(G)=k+1$ by Theorem 15 and $\mu(\bar{G}) \geqslant n-k-3$ by Corollary 13 .

\section{References}

[1] F. Barioli, W. Barrett, S. M. Fallat, H. T. Hall, L. Hogben, and H. van der Holst, On the graph complement conjecture for minimum rank, Linear Algebra Appl. 436 (2012), no. 12, 4373-4391, doi:10.1016/j.laa.2010.12.024.

[2] W. Barrett, S. M. Fallat, H. T. Hall, and L. Hogben, Note on Nordhaus-Gaddum problems for Colin de Verdière type parameters, Electron. J. Combin. 20 (2013), no. 3, Paper 56, 9.

[3] H. L. Bodlaender, A partial k-arboretum of graphs with bounded treewidth, Theoret. Comput. Sci. 209 (1998), no. 1-2, 1-45, doi:10.1016/S0304-3975(97)00228-4.

[4] Y. Colin de Verdière, Sur un nouvel invariant des graphes et un critère de planarité, J. Combin. Theory Ser. B 50 (1990), no. 1, 11-21, doi : 10.1016/0095-8956(90)90093-F (French).

[5] R. Diestel, Graph theory, 4th ed., Graduate Texts in Mathematics, vol. 173, Springer, Heidelberg, 2010.

[6] G. A. Dirac, On rigid circuit graphs, Abh. Math. Sem. Univ. Hamburg 25 (1961), $71-76$. 
[7] J. Ekstrand, C. Erickson, H. T. Hall, D. Hay, L. Hogben, R. Johnson, N. Kingsley, S. Osborne, T. Peters, J. Roat, A. Ross, D. D. Row, N. Warnberg, and M. Young, Positive semidefinite zero forcing, Linear Algebra Appl. 439 (2013), no. 7, 1862-1874, doi:10.1016/j.1aa.2013.05.020.

[8] S. Fallat and L. Mitchell, Colin de Verdière parameters of chordal graphs, Electron. J. Linear Algebra 26 (2013), no. 1, 49-62.

[9] F. Goldberg and A. Berman, On the Colin de Verdière number of graphs, Linear Algebra Appl. 434 (2011), no. 7, 1656-1662, doi:10.1016/j.laa.2010.05.008.

[10] L. Hogben, Nordhaus-Gaddum problems for Colin de Verdière type parameters, variants of tree-width, and related parameters, Recent Trends in Combinatorics, The IMA Volumes in Mathematics and its Applications, vol. 159, Springer International Publishing, Switzerland, 2015.

[11] I. Izmestiev, The Colin de Verdière number and graphs of polytopes, Israel J. Math. 178 (2010), 427-444, doi:10.1007/s11856-010-0070-5.

[12] G. Joret and D. R. Wood, Nordhaus-Gaddum for treewidth, European J. Combin. 33 (2012), no. 4, 488-490, doi:10.1016/j.ejc.2011.10.005. MR2864434 (2012k:05195)

[13] R. J. Kang, L. Lovász, T. Müller, and E. R. Scheinerman, Dot product representations of planar graphs, Electron. J. Combin. 18 (2011), no. 1, Paper 216, 14.

[14] E. Koch and M. A. Perles, Covering efficiency of trees and $k$-trees, Proceedings of the Seventh Southeastern Conference on Combinatorics, Graph Theory, and Computing (Louisiana State Univ., Baton Rouge, La., 1976), Utilitas Math., Winnipeg, Man., 1976, pp. 391-420. Congressus Numerantium, No. XVII.

[15] A. Kotlov, L. Lovász, and S. Vempala, The Colin de Verdière number and sphere representations of a graph, Combinatorica 17 (1997), no. 4, 483-521, doi:10.1007/BF01195002.

[16] L. Lovász, Steinitz representations of polyhedra and the Colin de Verdière number, J. Combin. Theory Ser. B 82 (2001), no. 2, 223-236, doi:10.1006/jctb.2000.2027.

[17] _ The Colin de Verdière number of a graph, 2007. Available online at http: //www.cs.elte.hu/ lovasz/colinnotes.pdf.

[18] _ On the Shannon capacity of a graph, IEEE Trans. Inform. Theory 25 (1979), no. 1, 1-7, doi:10.1109/TIT.1979.1055985.

[19] Geometric representations of graphs, 2009. Available online at http://www . cs.elte.hu/ lovasz/geomrep.pdf.

[20] L. Lovász and K. Vesztergombi, Geometric representations of graphs, Paul Erdős and his mathematics, II (Budapest, 1999), Bolyai Soc. Math. Stud., vol. 11, János Bolyai Math. Soc., Budapest, 2002, pp. 471-498. 
[21] L. H. Mitchell, On the graph complement conjecture for minimum semidefinite rank, Linear Algebra Appl. 435 (2011), no. 6, 1311-1314, doi:10.1016/j.1aa.2011.03.011.

[22] E. A. Nordhaus and J. W. Gaddum, On complementary graphs, Amer. Math. Monthly 63 (1956), 175-177.

[23] T. D. Parsons and T. Pisanski, Vector representations of graphs, Discrete Math. 78 (1989), no. 1-2, 143-154, doi:10.1016/0012-365X (89) 90171-4.

[24] H. P. Patil, On the structure of k-trees, J. Combin. Inform. System Sci. 11 (1986), no. $2-4,57-64$.

[25] D.J. Rose, On simple characterizations of k-trees, Discrete Math. 7 (1974), 317-322.

[26] J. Sinkovic and H. van der Holst, The minimum semidefinite rank of the complement of partial k-trees, Linear Algebra Appl. 434 (2011), no. 6, 1468-1474, doi:10.1016/j.1aa.2010.11.013.

[27] A. Takahashi, S. Ueno, and Y. Kajitani, Minimal acyclic forbidden minors for the family of graphs with bounded path-width, Discrete Math. 127 (1994), no. 1-3, 293304, doi:10.1016/0012-365X (94)90092-2. Graph theory and applications (Hakone, 1990).

[28] H. van der Holst, L. Lovász, and A. Schrijver, The Colin de Verdière graph parameter, Graph theory and combinatorial biology (Balatonlelle, 1996), Bolyai Soc. Math. Stud., vol. 7, János Bolyai Math. Soc., Budapest, 1999, pp. 29-85. 